\section{Andreas Ruhrmann}

Xin Zhang

Aeronautics and Astronautics,

School of Engineering Sciences,

University of Southampton,

Southampton S017 1BJ, UK
Influence of Diffuser Angle on a Bluff Body in Ground Effect

The forces and pressures on a generic bluff body in ground effect were investigated. The bluff-body model was equipped with interchangeable underbody diffuser ramps and side plates. Five different diffuser angles were tested: 5, 10, 15, 17, and 20 deg to the horizontal. The experiments were undertaken in a low-speed wind tunnel equipped with a moving ground. Load cells, pressure taps, and surface flow visualization were the techniques used to evaluate the flow field. The flow field is characterized by vortex flow and three-dimensional flow separation. A region of hysteresis was found for the 15, 17, and 20 deg diffusers. As the ride height is varied, five different flow types can be identified with three subtypes within the region of hysteresis. The force reduction phenomenon was found to be caused by both vortex breakdown and flow separation. [DOI: 10.1115/1.1537252]

\section{Introduction}

A symmetrical body in ground effect will generate aerodynamic force pointed to the ground (downforce). The flow between the body and the ground is accelerated leading to reduced pressure. Introducing camber to the body in the form of an upswept ramp at the rear creates a diffuser between the ground and the body. Since the diffuser exits to the base pressure of the model, the flow under the model accelerates to a greater degree than without the diffuser. This increase in downforce is accompanied by complex flow physics within the diffuser. The upsweep initiates threedimensional flow separation which leads to vortex formation and possible flow reversal. This area of research is especially important in the automobile industry with respect to performance and safety. A complete understanding of the complex fluid flow physics is essential for the development of flow control measures.

Currently there is only limited research published in the field of diffuser flow in ground effect, [1-6]. The general principle of how a diffuser functions in ground effect is described by Sovran [3]. It is likened to a venturi, with the flow rate under the model governed mainly by the efficiency of the diffuser. As the diffuser efficiency is increased, the flow rate increases and the static pressure under the model drops leading to a rise in the level of downforce. The study completed by Cooper et al. [4] found that as the ride height is reduced down to a critical ride height, the downforce increases. Below the critical ride height, the downforce reduces rapidly. It was surmised that at this ride height, the ground and model boundary layers form a substantial proportion of the flow into the diffuser. Senior and Zhang [6] found that for a bluff body with a 17-deg diffuser, the rapid reduction in downforce was not due to the increased influence of the boundary layers as changes in the Reynolds number did not influence the critical ride height. They also found that one of the two counterrotating vortices that form in the diffuser disappears below the critical ride height resulting in an asymmetric flow pattern with flow reversal on one side. Four different types of force behavior were identified through a range of ride heights.

The goals of the present research is to further the understanding of the complex three-dimensional flow field to aid the development of improved diffuser design and methods of flow control. To aid in the understanding of the complex flow phenomena, several different diffuser ramp angles were studied to investigate the effects of area ratio, pressure gradient and ground proximity. The diffuser ramp angles investigated were 5, 10, 15, 17, and 20 deg to the horizontal. The various angles are tested to establish a data-

Contributed by the Fluids Engineering Division for publication in the JOURNAL OF FLUIDS ENGINEERING. Manuscript received by the Fluids Engineering Division July 16, 2001; revised manuscript received October 7, 2002. Associate Editors: J. Bridges and J. Katz. base of results for comparison with computational fluid dynamics and from which flow control methods could be developed. The range of angles includes lower angles (5 and $10 \mathrm{deg}$ ) where no flow separation occurs in two dimensional planar diffusers. These will hopefully show the influence of the separation bubble on the force-ride height characteristics of the diffusers with larger divergence angles.

\section{Experimental Setup}

The experiments were undertaken at the University of Southampton in the low-speed closed-circuit wind tunnel with test section dimensions of $2.1 \mathrm{~m} \times 1.5 \mathrm{~m}$. The wind tunnel is equipped with a moving ground measuring $3.5 \mathrm{~m}$ in length and $1.5 \mathrm{~m}$ in width. The experiments were run at a wind and ground speed of $30 \mathrm{~m} / \mathrm{s}$ at a freestream turbulence level of $0.2 \%$. There is a boundary layer removal system ahead of the moving ground. It consists of a slot through which the boundary layer passes and a perforated plate just ahead of the belt (moving ground) through which the remainder of the boundary layer is sucked. The velocity reaches freestream values $2 \mathrm{~mm}$ above the belt. Further details can be found in Senior [7].

A schematic of the wind tunnel setup is shown in Fig. 1. It is mounted on a motorized main strut and tail wire (a free pivoting, thin carbon strut) configuration. The motorized struts are each powered by a stepper motor allowing the model to be moved up to a maximum ride height of $180 \mathrm{~mm}$. The lowest ride height is constrained by a minimum safety clearance above the moving ground.

The bluff body of the model is constructed of carbon fiber. Using different diffuser ramps, the angle of the diffuser can be varied. The ramps are constructed of $3-\mathrm{mm}$ steel sheets. The angles tested are 5, 10, 15, 17, and $20 \mathrm{deg}$. This range of angles was chosen to create a better understanding of different flow physics which occur at the different ride heights. By varying the diffuser angle, the significance of the blockage between the ground and the model underbody can be investigated as the various flow types will occur at a different ride height for each diffuser angle. Transition on the model is fixed using a $0.4-\mathrm{mm}$ diameter wire placed at $100 \mathrm{~mm}$ from the nose of the model.

The tests conducted include force measurements, surface pressure measurements, and oil-streak flow visualisation. The forces are measured using a two component load cell (vertical and horizontal forces) on the base of the main strut and a single component load cell (vertical force) on the top of the tail wire. A total of 186 pressure taps are arranged on the underside and base of the model to give both centerline pressures and spanwise measure- 


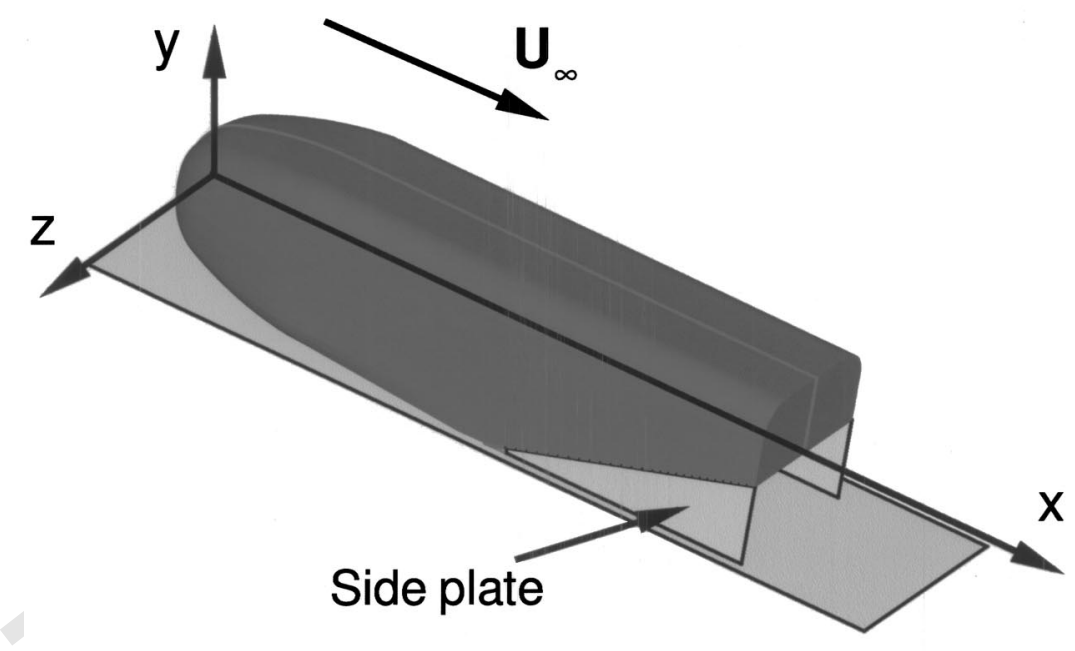

(a)

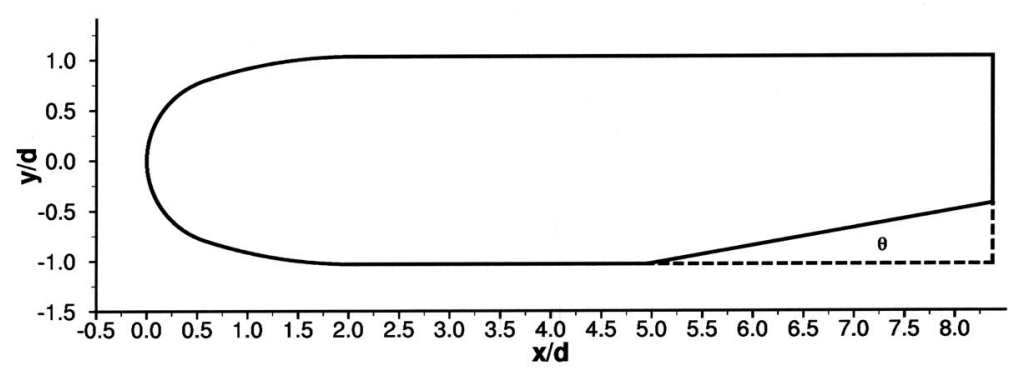

(b)

Fig. 1 Schematic of model; (a) a prospective view of the model, (b) a side view of the model

ments. The pressures were measured using a zero operate calibrate (ZOC) system. A mixture of titanium dioxide and light oil was used to visualize the surface streak lines.

Applying the procedure described by Moffat [8] the singlesample uncertainties in the experiment were calculated. The ride height was kept to within $\pm 0.1 \mathrm{~mm}$ and the yaw angle was set to $\pm 0.05 \mathrm{deg}$. The forces were averaged over 15 seconds at each ride height, with a one-second delay between ride height changes. The pressures on the ZOC were sampled for ten seconds on each of the two banks, also with a one second delay between ride height change. For the 20-deg diffuser at $90-\mathrm{mm}$ ride height, the uncertainties in the $C_{L}$ and $C_{D}$ measurements are \pm 0.0016 and \pm 0.0008 , respectively. The uncertainty in the pressure measurements in the previous configuration for a $C_{P}$ of -2.0 is \pm 0.0188 . This uncertainty incorporates the quoted accuracy of the equipment.

\section{Results and Discussion}

Definition of Flow Regimes. The downforce and drag curves in Fig. 2 demonstrate that there are two different types of flow regimes dependent on the diffuser angle. The curves for the 15, 17 , and $20 \mathrm{deg}$ (high angle) diffusers have similar characteristics as do the 5 and 10 deg (low angle) diffusers. The 10-deg diffuser appears to be more of a transition angle between the two types as it contains characteristics of both. As the height above the moving ground is varied, the slopes of the curves change indicating changes in the flow physics. The high angle diffusers demonstrate the same characteristics as found by Senior and Zhang [6]. Utilizing the moving strut, one additional flow type along with extensions to three of the existing flow types in the region of hysteresis were identified. All flow types are described in Table 1. The different flow regions are shown on the downforce curve for the 15-deg diffuser in Fig. 3. Starting the wind tunnel with the model at a fixed height within the region of hysteresis, the flow always reverted to the curve of lower downforce. The high downforce portion of the hysteresis loop was found to be unstable, as any disturbances would trigger it to fall onto the low downforce curve. During the 15-second sampling period of the forces, a real time display of the measured forces indicated that the flow was unsteady in this region. The real time display of the measured forces suggested that most of region $\mathrm{b}$ and all of regions $\mathrm{c}, \mathrm{d}, \mathrm{d}^{\prime}$, and $\mathrm{e}$ were unsteady as well.

Comparing the downforce curves of the low angle diffusers to those of the high angle diffusers, distinctions between the two sets are identifiable. Although the curves are similar, the closer proximity to the ground introduces differences. There is no hysteresis loop and the sudden reduction in downforce is not as pronounced. Type a and b flow still exist, however, there is a pronounced increase in downforce through the lower portion of region $b$. This is clearest for the 5-deg diffuser. Type $\mathrm{c}$ and d flow exist only to a very limited extent for the 10 -deg diffuser. Due to the lower ride heights, it is assumed that both the underbody and ground boundary layers form a considerable proportion of the flow entering the diffuser at these ride heights, causing the direct transition into type 


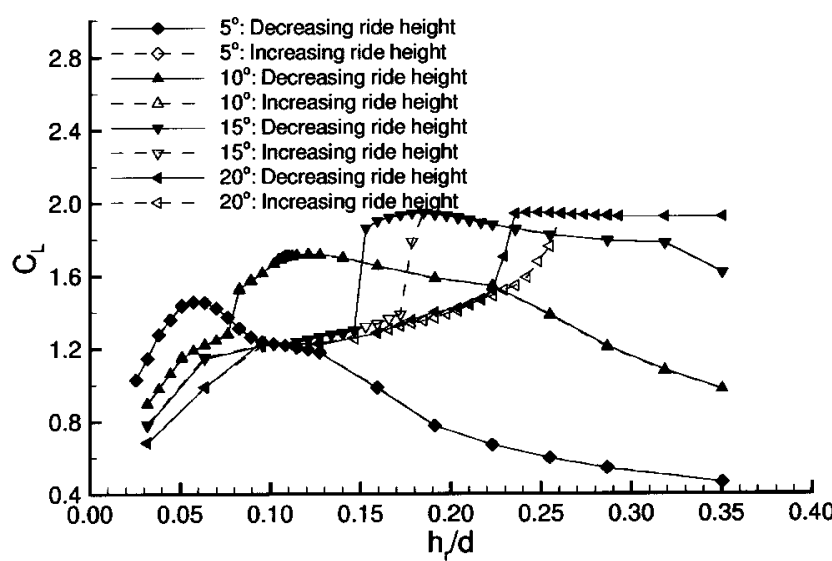

(a)

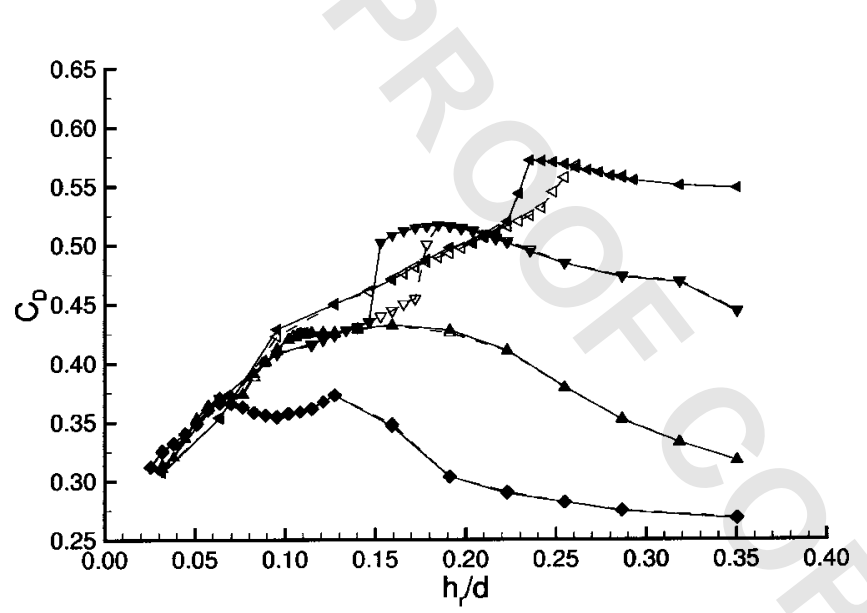

(b)

Fig. 2 Force curves: $30 \mathrm{~m} / \mathrm{s}$; (a) downforce coefficient, (b) drag coefficient

e flow. The underbody boundary layer is predicted to be (O) $0.096 d$ from flat plate theory, although this value will certainly change with the favorable pressure gradient and increased flow velocity under the model. Measurements by Senior [7] using laser doppler anemometry supports our assertion.

Maximum Downforce. Reducing the normalized ride height with the diffuser angle, it becomes apparent that the maximum downforce occurs at similar values of $h_{r} /(d \times \theta)$ (Fig. 4), where $\theta$ is the divergence angle of the diffuser in radians. The maximum

Table 1 Flow types in high angle diffusers

\begin{tabular}{|c|c|c|}
\hline Type & Direction & Description (see Fig. 3) \\
\hline $\mathrm{a}$ & both & increasing downforce with reducing ride height \\
\hline $\mathrm{b}$ & both & $\begin{array}{l}\text { almost constant region high downforce, slight increas } \\
\text { with reducing ride height }\end{array}$ \\
\hline $\mathrm{b}^{\prime}$ & down & $\begin{array}{l}\text { an extension of } \mathrm{b} \text { when the ride height is } \\
\text { decreasing, downforce starts to reduce }\end{array}$ \\
\hline $\mathrm{c}$ & up & rapid increase in downforce with ride height \\
\hline$c^{\prime}$ & down & $\begin{array}{l}\text { rapid downforce reduction with a small decrement } \\
\text { in ride height }\end{array}$ \\
\hline $\mathrm{d}$ & both & an almost flat region of low downforce \\
\hline$d^{\prime}$ & up & $\begin{array}{l}\text { an extension of } \mathrm{d} \text { when the ride height is increasing, } \\
\text { slow increase in downforce }\end{array}$ \\
\hline e & both & a further reduction in downforce at flow ride heights \\
\hline \multicolumn{3}{|c|}{ Regions $\mathrm{b}^{\prime}$ and $\mathrm{c}$ overlap (in terms of ride heights), as well as $\mathrm{d}^{\prime}$ and $\mathrm{c}^{\prime}$} \\
\hline
\end{tabular}

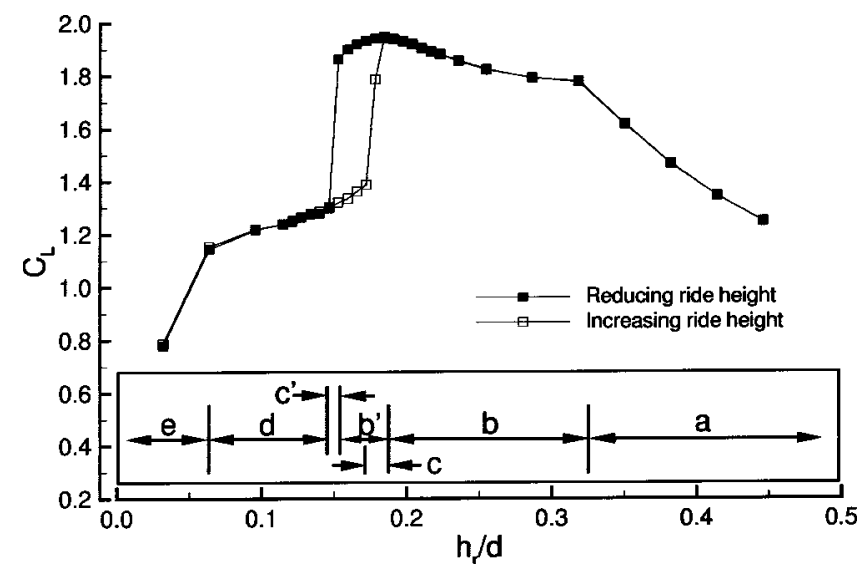

Fig. 3 Flow types for high angle diffusers: 15 deg diffuser, $C_{L}$, $30 \mathrm{~m} / \mathrm{s}$

occurs at approximately $0.7 h_{r} /(d \times \theta)$. Using this, the diffuser angle (or length) could be optimised with regard to expected ride heights.

Flow visualization on the ramp surfaces taken at maximum downforce as shown in Fig. 5, demonstrate some of the differences between the low and high angle diffusers. There is no separation bubble on the 5-deg ramp, although towards the end of the diffuser, the flow appears to be slow and unsteady. The flow on the 10-deg diffuser ramp demonstrates a closed separation bubble. The bubble forms somewhat downstream of the inlet and closes just ahead of the diffuser exit. Upstream of this point, the vortices appear to become wider, with a reduced swirl indicating the onset of vortex breakdown. The open separation bubble forming on the 20-deg diffuser ramp is very similar to those found on the 15 and 17 deg diffuser ramps. From the surface flow patterns downstream of the primary separation line, there appears to be only a small region where the flow is reversed. The separated flow is entrained into the vortices reducing the axial momentum. The reduced swirl of the vortices downstream of the primary separation line are an indication of vortex breakdown. In comparison to the the 10-deg diffuser, the primary flow separation line is closer to the inlet at the center of the ramp. For the 15 and 17 deg diffuser ramps, the primary flow separation line moves closer to the inlet below the maximum downforce ride height up to the point where the flow becomes asymmetrical.

Variation of Ride Height. At larger ride heights (type a flow), the adverse pressure gradient along the diffuser centerline is relatively weak (Fig. 6). The ratio of exit area to inlet area is low.

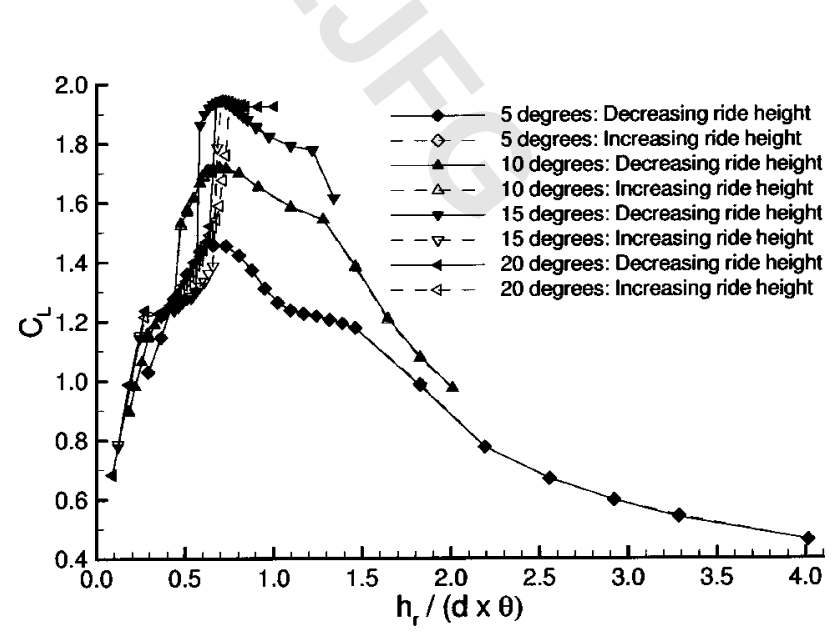

Fig. 4 Downforce coefficients: renormed ride heights, $30 \mathrm{~m} / \mathrm{s}$ 


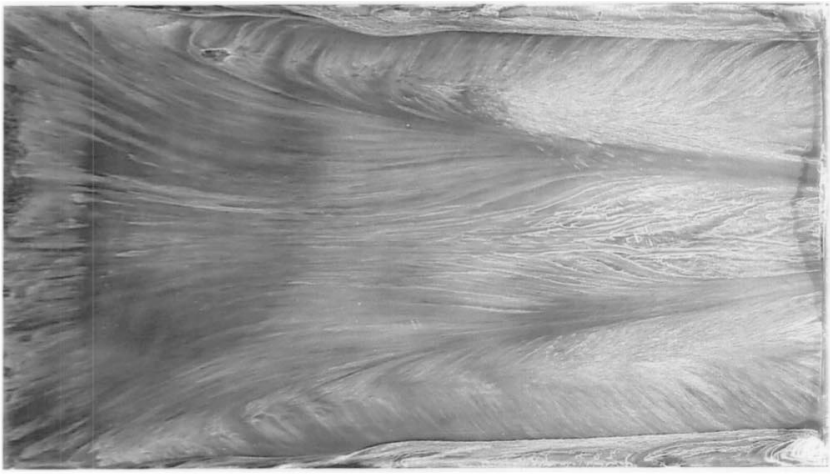

(a)

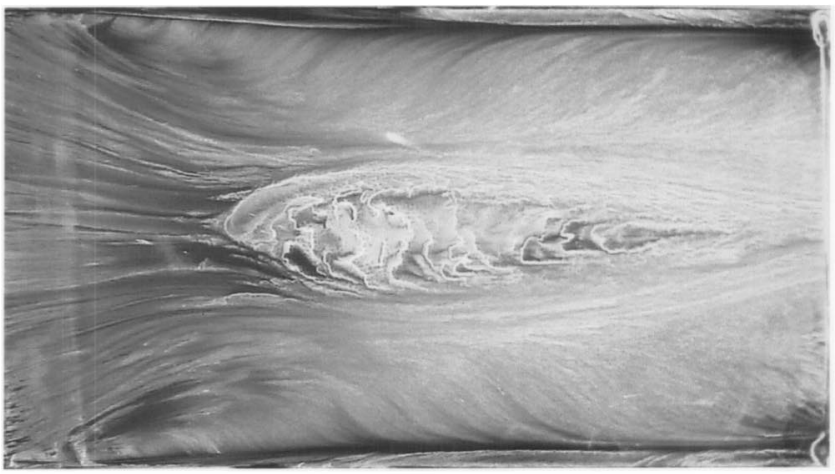

(b)

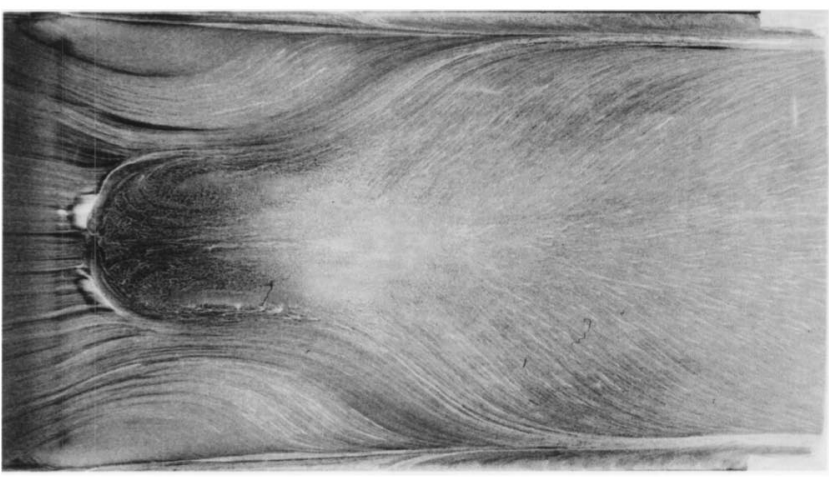

(c)

Fig. 5 Surface flow visualization on the ramp at maximum downforce, $30 \mathrm{~m} / \mathrm{s}$. Flow from left to right. Picture area corresponds to the ramp area. (a) 5-deg diffuser: $h_{r} /(d \times \theta)=0.657$, (b) 10-deg diffuser $h_{r} /(d \times \theta)=0.694$, (c) 15-deg diffusermoving down: $h_{r} /(d \times \theta)=0.584$.

No quasi-two-dimensional flow separation occurs in the center of the diffuser ramps. As the ride height is reduced through this region of type a flow, the area ratio (ratio of exit to inlet area of the diffuser) increases along with the effect of moving closer to the ground. The flow speed under the model rises (reduced static pressure, see Fig. 6(a)) resulting in increased downforce. The flow is dominated by two counter rotating vortices which prevent or limit the formation of a separation bubble. The pressure at the inlet of the diffuser reduces with ride height. Low pressure regions form in the corners of the diffuser where the vortices originate (Fig. 7). Figure 8 shows that the average base pressure of the model remains fairly constant throughout this region, although for the 5-deg diffuser, the base pressure begins to drop at a higher ride height $\left(h_{r} /(d \times \theta)=2.2\right)$ indicating a change in the flow physics.

Reducing the ride height further in region $b$ for the large angle diffusers, the flow field starts to change. The average base pres-

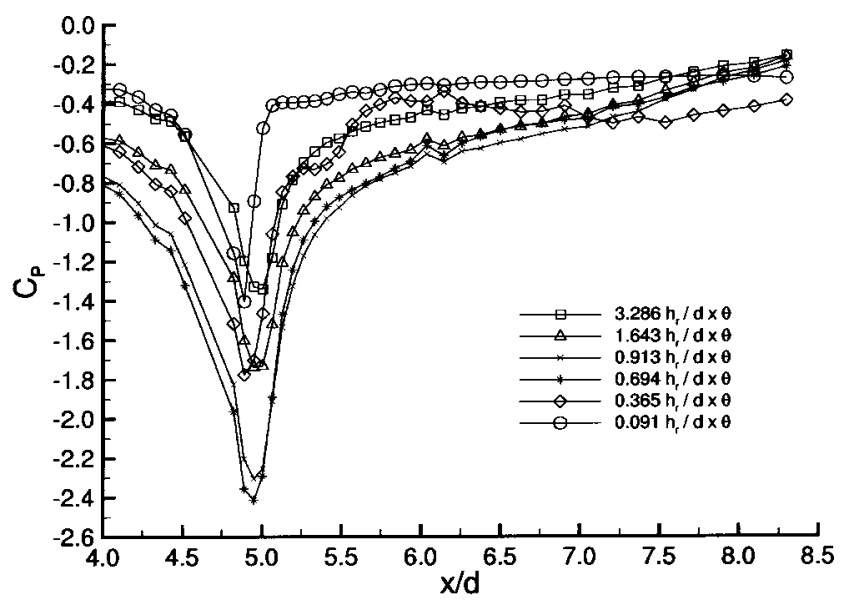

(a)

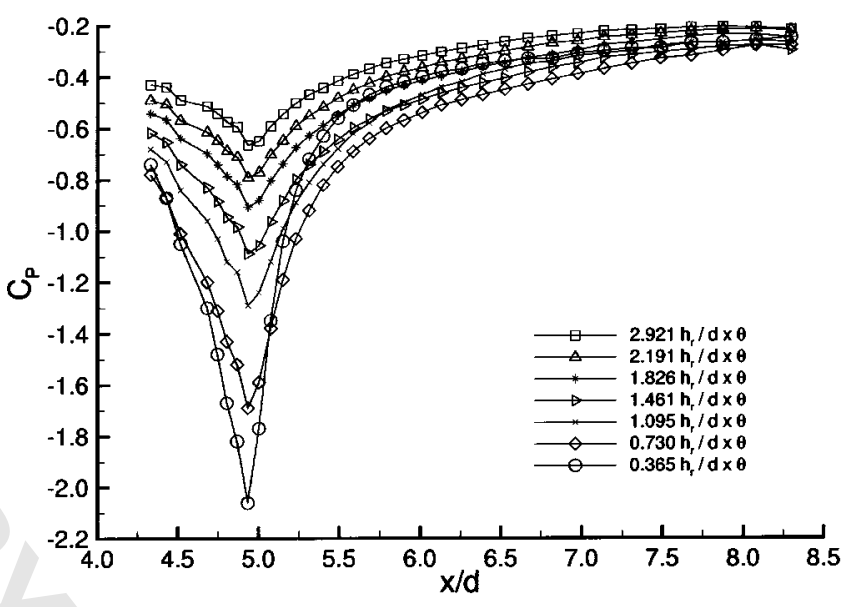

(b)

Fig. 6 Centerline pressure coefficients: $30 \mathrm{~m} / \mathrm{s}$. (a) 20 deg diffuser, (b) 5 deg diffuser.

sure of the model drops quickly (Fig. 8(a)) indicating a loss of pressure recovery within the diffuser. Drawing a parallel to twodimensional planar diffusers, a reduction in pressure recovery is generally associated with the onset of flow separation. In the case of the high angled diffusers, this is the case. The flow starts to separate at the inlet in the center of the diffuser. This separation bubble ceases to exist downstream as the vortices grow in diam-

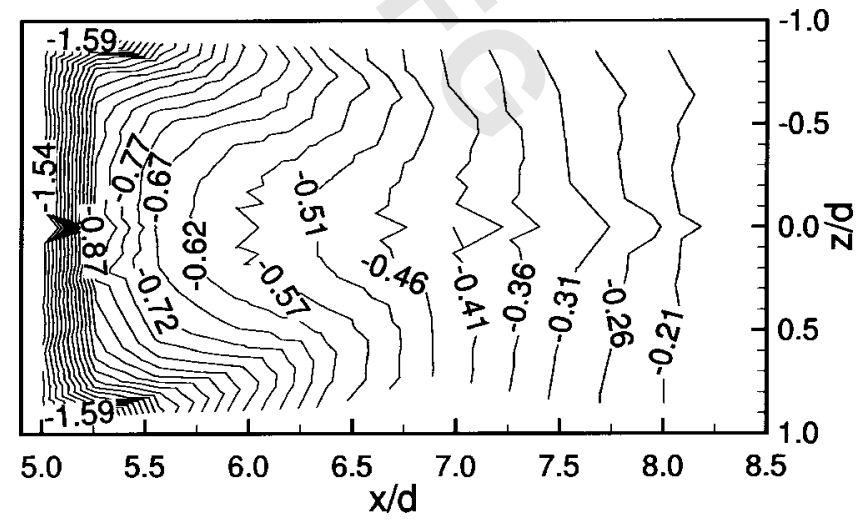

Fig. 7 Surface pressure coefficients: 20 degree diffuser, $h_{r} /(d \times \theta)=2.008,30 \mathrm{~m} / \mathrm{s}$ 


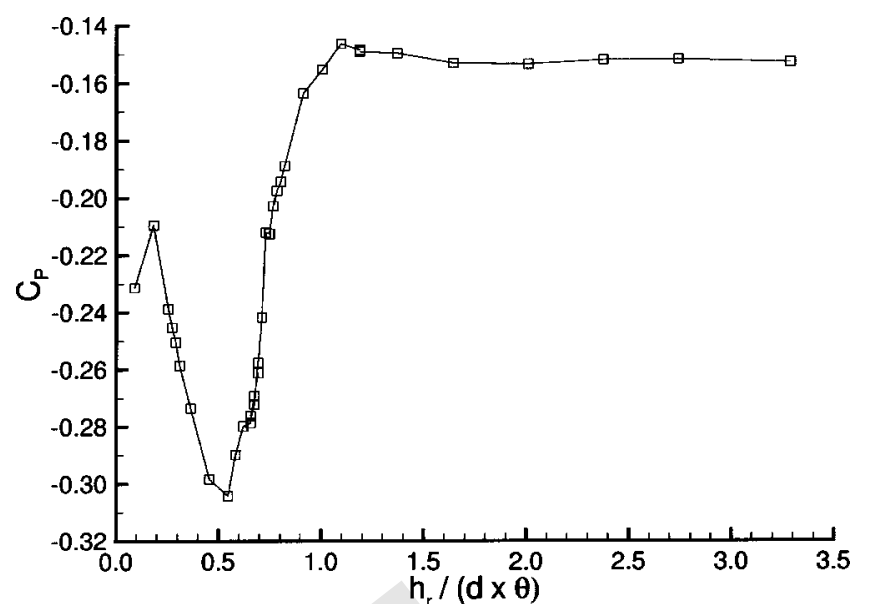

(a)

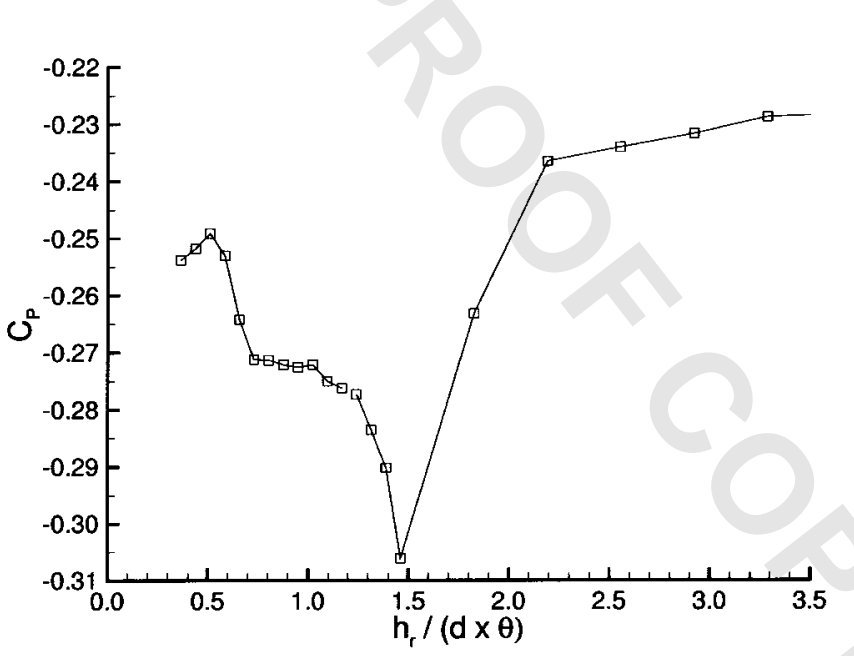

(b)

Fig. 8 Mean base pressures, $30 \mathrm{~m} / \mathrm{s}$; (a) 20 deg diffuser, (b) 5 deg diffuser

eter. However, in the case of the 5-deg diffuser, no such flow separation is apparent. The cause of this reduction in pressure recovery may be due to the onset of vortex breakdown. As a vortex begins to breakdown, the axial velocity of the core reduces. This effectively reduces the area ratio of the diffuser as the lower speed in the cores of the vortices essentially decreases the exit area of the diffuser. The velocity of the air between the vortices down the centerline appears to increase as the centerline pressure coefficients along the length of the diffuser ramp reduce (Fig. $6(b)$ when moving from an $h_{r} /(d \times \theta)$ value of 1.095 to 0.730 . The second set of vortices present at $1.241 h_{r} /(d \times \theta)$ at approximately $z / d= \pm 0.4$ towards the end of the diffuser diminish in strength to $0.730 h_{r} /(d \times \theta)$ (Fig. 9). The vortices at the edge of the ramp increase in strength.

In the case of the 5-deg diffuser, the minimum base pressure occurs at the onset of type $b$ flow. The peak in the drag curve at this point is due to the large base area of the model in this configuration. The lower diffuser angle and ride heights limit the strength and size of the vortices in the diffuser resulting in lower induced drag relative to the higher angled diffusers, as a result, the pressure drag becomes increasingly significant. An increase of base pressure beyond this point indicates that the boundary layers entering the diffuser are beginning to influence the flow to a larger degree. Whereas with the 17-deg diffuser the base pressure reduces to a minimum at the point where the hysteresis loop closes at the lower ride height, the base pressure for the 5-deg diffuser

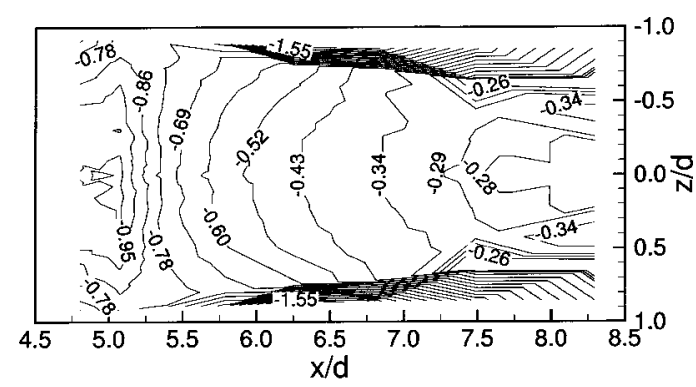

(a)

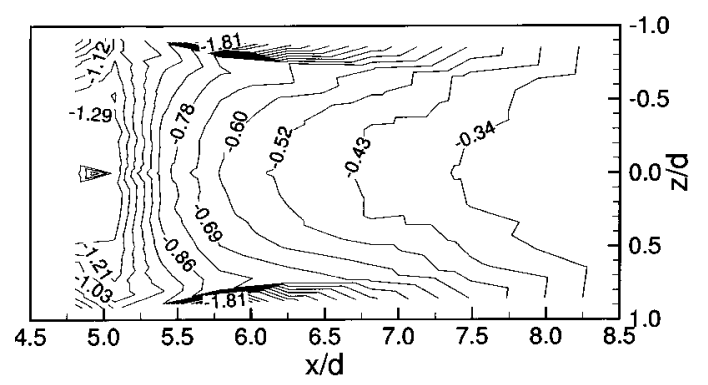

(b)

Fig. 9 Pressure contours: 5 deg diffuser, $30 \mathrm{~m} / \mathrm{s}$; (a) $h_{r} /(d$ $\times \theta)=1.241,(b) h_{r} /(d \times \theta)=0.730$

increases beyond the maximum downforce. As the boundary layers make up an increasing proportion of the diffuser inlet, the flow rate under the model decreases reducing the importance of the diffuser, moving towards the limiting case of zero ride height where no air passes under the model or through the diffuser.

The vortex observed in the current study is similar in characters to the leading edge vortex studied by Polhamus [9] using a leading-edge suction analogy and by Wentz and Kohlman [10] in a wind tunnel model test. In Fig. 10 evidence of vortex breakdown can be seen. As described in an earlier work of Lambourne and Bryer [11], the surface streaklines change downstream of the vortex bursting point. The swirl becomes less evident and the vortex becomes wider. This may also be a contributing factor to the ensuing asymmetry of the flow at lower ride heights. The apparent unsteadiness of the flow field may result in the bursting point of one vortex to move further upstream momentarily, causing a disturbance to the pressure field resulting in the asymmetric flow of region $\mathrm{d}$.

The importance of the separation bubble in the center of the diffuser inlet can be seen in Fig. 10. Just before the hysteresis loop

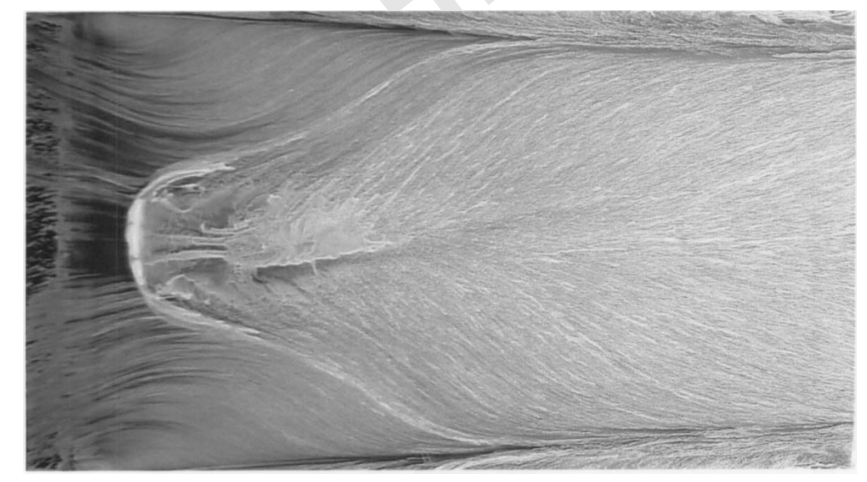

Fig. 10 Surface flow visualization on the ramp: 20 deg diffuser, $h_{r} /(d \times \theta)=0.694,30 \mathrm{~m} / \mathrm{s}$. Flow from left to right. Picture area corresponds to the ramp area. 


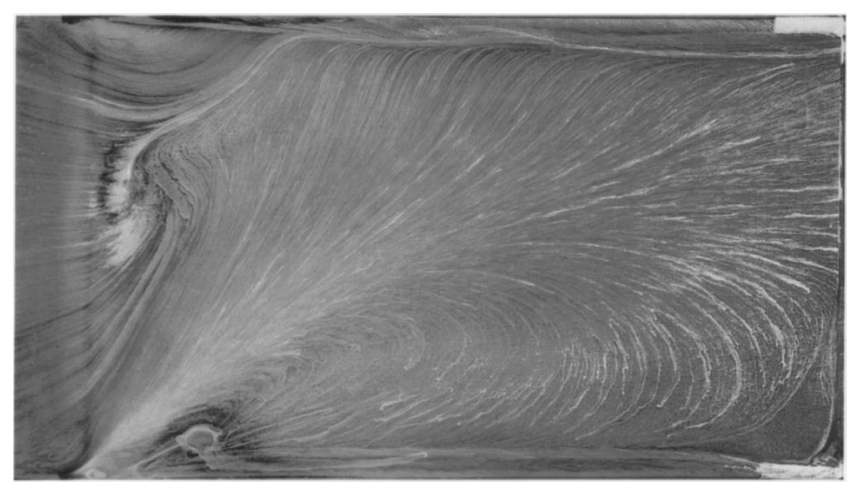

Fig. 11 Surface flow visualization on the ramp: 15 deg diffuser, $h_{r} /(d \times \theta)=0.560,30 \mathrm{~m} / \mathrm{s}$. Flow from left to right. Picture area corresponds to the ramp area.

closes, at the lower end of region $b^{\prime}$, the separation bubble has almost reached the inlet of the diffuser. At the point where the hysteresis loop is closed at the lower end moving through region $\mathrm{c}^{\prime}$, the flow becomes asymetrical as shown in Fig. 11. Once the separation point reaches the inlet it cannot travel further upstream due to the favorable pressure gradient ahead of the inlet. Due to the extremely low pressures to either side of the inlet (Fig. 12), any disturbances will cause the large asymmetry in the flow to occur. The separation bubble gets swept to one side and a large recirculation region forms.

The direction of the asymmetry occurred randomly, but once established did not switch sides. This gives further indication that the type $b$ flow is unstable. As found in experiments with high speed flows over axisymmetric bodies, any slight imperfection on the surface can cause asymmetries in the vortex flow field. As slight movement of the model may trigger the asymmetry in the flow field to take one direction over the other.

Progressing downwards through region $\mathrm{d}$, the focus point close to the inlet of the diffuser on the side of the recirculating region (Fig. 11) moves downstream and towards the center of the diffuser. The region of recirculating flow is easily identifiable in the pressure contour plot of the 20-deg diffuser within type d flow in Fig. 13 as the large region of constant pressure. The pressure coefficients indicate that the remaining longitudinal vortex is not as strong as those at higher ride heights.

Flow type e is characterized by a steepening of the downforce curve. For the large angle diffusers this appears to occur when the ride height reaches the order of magnitude of the expected boundary layer thickness. From the pressure coefficient contour plot in Fig. 14 at a ride height of $0.091 h_{r} /(d \times \theta)$ it is clear that the flow is separated over the majority of the diffuser ramp. The slight asymmetry in the contours is caused by a weak vortex forming on one side of the inlet. The centerline pressures from Fig. 6(a) show that the pressure spike is reduced dramatically and occurs

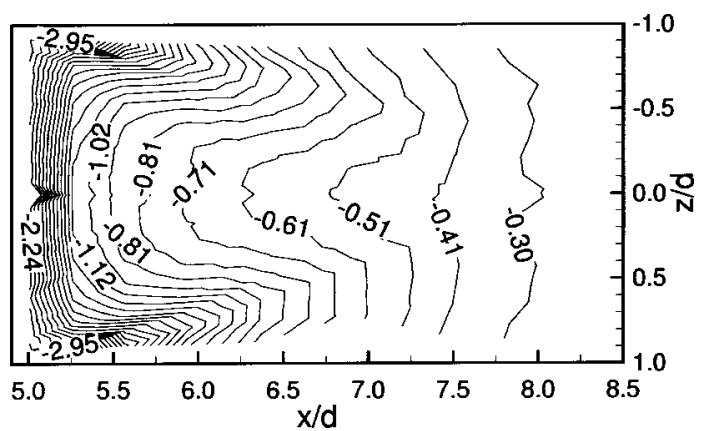

Fig. 12 Surface pressure coefficients: 20 deg diffuser, $h_{r} /(d$ $\times \theta)=0.694,30 \mathrm{~m} / \mathrm{s}$

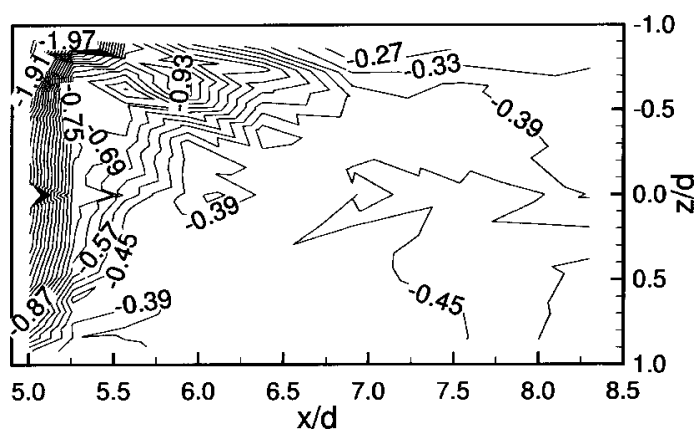

Fig. 13 Surface pressure coefficients: 20 deg diffuser, $h_{r} /(d$ $\times \theta)=0.365,30 \mathrm{~m} / \mathrm{s}$

further upstream. This indicates that there is not enough energy in the flow to overcome the adverse pressure gradient in the diffuser.

\section{Further Discussion}

The plot of drag variation with ride height for the 5 and $10 \mathrm{deg}$ diffusers (Fig. 2(b) show two peaks, although the trough is better defined for the 5-deg diffuser. As can be seen with the pressure plots from the 5-deg diffuser in Fig. 9, at 1.241 $h_{r} /(d \times \theta)$ or $0.108 h_{r} / d$ there is a second set of vortices present, inboard of the first. These are no longer present on the pressure plot at $0.730 h_{r} /(d \times \theta)$ or $0.064 h_{r} / d$. The ouboard votices appear to increase in strength as the minimum $C_{P}$ drops with reducing ride height. Between these two ride heights, the centerline pressure coefficients (Fig. 6(b)) also drop along the length of the diffuser, with a larger difference at the inlet and approaching the same value at the exit. Figure $8(b)$ shows an increase in the mean base pressure between these ride heights. The weakening of the inboard vortices, and the ensuing increase of the axial velocity through the diffuser cause an increase in the downforce. As the inboard vortices burst, the initial increase in downforce due to the strengthening of the outboard vortices is gradual. As the ride height is reduced, the flow through the diffuser becomes increasingly twodimensional in nature as the aspect ratio $\left(2 d / h_{r}\right)$ of the inlet increases to a value of 31.4 at $0.730 h_{r} /(d \times \theta)$. The expansion of the axial flow region through the center of the diffuser improves the pressure recovery of the diffuser, creating the peak in the downforce at $0.730 h_{r} /(d \times \theta)$. It is surmized that the dip in the drag curve is caused by the increasing base pressure and by a reduction in the induced drag due to the diminishment of the inboard vortices. The increase in drag below the trough is most likely caused by the increase of strength in the outboard vortices and the accompanying increase of induced drag. The base pressure in this region does not increase as rapidly as when the the drag reduces.

A map of diffuser performance, Fig. 15, shows the different operating regions of the different diffusers angle with respect to

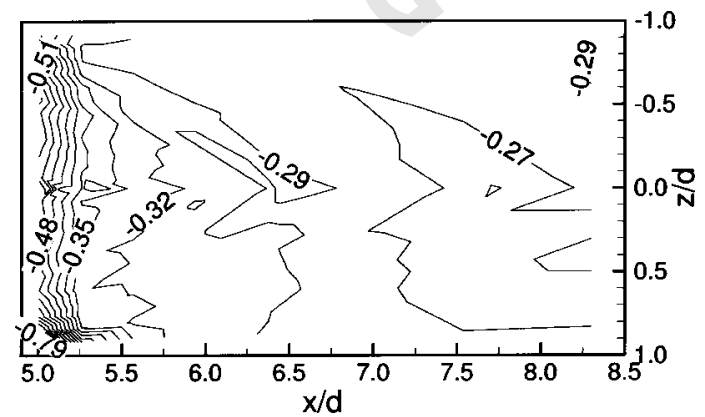

Fig. 14 Surface pressure coefficients: 20 deg diffuser, $h_{r} /(d$ $\times \theta)=0.091,30 \mathrm{~m} / \mathrm{s}$ 


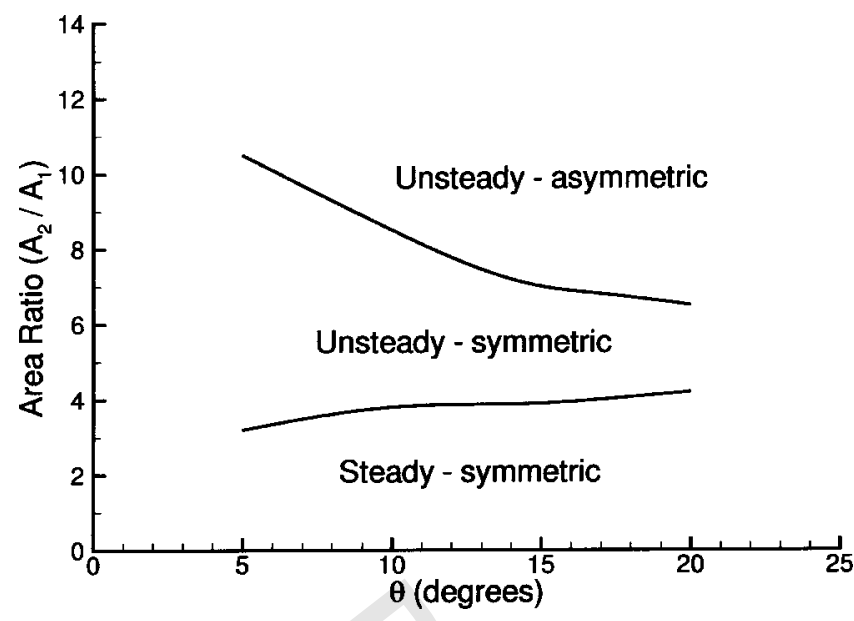

Fig. 15 Different flow regimes

area ratio. The shift from steady symmetric flow (type a) to unsteady and symmetric (type b) occurs as similar area ratios for all diffuser ramp angles, indicating that it is primarily governed by the adverse pressure gradient. The narrowing of the unsteadysymmetric region towards the larger diffuser angles indicates that the streamwise adverse pressure gradient along the diffuser is not the driving factor for the transition to asymmetric flow. From the negative slope of this boundary it is clear that the underbody and ground boundary layers are not an important aspect of this transition. If the boundary layers were an influencing factor, the shift would occur at lower area ratios for the lower diffuser ramp angles as the effective inlet area would be reduced. Supporting evidence of the above observation can also be found in the wing in ground study of Zerihan and Zhang [12] and the high angle diffuser study of Senior and Zhang [6], where fixed ground tests were also performed. We have also performed fixed ground tests for all the angles. These fixed ground tests produced essentially the same observations. The effect of a fixed ground was first pointed by Burgen et al. [13] using, among other things, a bluff body in ground effect. The use of a fixed ground generally results in a downward shift in the downforce curve because of an effective reduction on mass flow rate between the ground and the model. An additional factor is the reduction in the effective ground height due to the displacement effect of the boundary layers. A change in the fundamental force characteristics does not occur above flow region e.

\section{Summary}

1 It is surmized that for the lower angled diffusers, vortex breakdown is the primary cause of force reduction. A combination of flow separation and vortex breakdown causes the force reduction for the high angle diffusers.

2 The underbody boundary layers do not appear to change the force characteristics of the diffuser at ride heights above region e, the onset of which approximately corresponds to the estimated boundary layer thickness. This occurs at a much larger ride height for the fixed ground case, [6].

3 A region of hysteresis occurs over the force reduction region for the 15,17 , and 20 degree diffusers.
4 The flow through the low angled diffusers is influenced by the underbody, and presumeably ground, boundary layers. Especially the formation and breakdown of the vortices.

5 The flow is unsteady in regions b, c, d, and e.

\section{Acknowledgments}

Andreas Ruhrmann wishes to thank British American Racing for providing a scholarship. The authors wish to thank Willem Toet and Andrea Senior for their help and suggestions.

\section{Nomenclature}

$C_{D}=$ drag coefficient, $D / q_{\infty} S$

$C_{L}=$ downforce coefficient, $L / q_{\infty} S$

$C_{P}=$ pressure coefficient, $\left(p-p_{\infty}\right) / q_{\infty}$

$D=$ drag

$L=$ downforce

$M=$ pitching moment

$p=$ static pressure

$p_{\infty}=$ freestream static pressure

$q_{\infty}=$ freestream dynamic pressure, $\rho_{\infty} U_{\infty}^{2} / 2$

$U_{\infty}=$ freestream velocity

$\operatorname{Re}=$ Reynolds number, $\rho_{\infty} U_{\infty} l / \mu$

$S=$ model frontal area

$d=$ model half-width measured in $y$-direction

$h_{r}=$ model ride height

$l=$ length of model

$x, y$,

$z=$ cartesian coordinates: $x+$ ve downstream, $y+$ ve up, $z$ + ve to port

$\rho_{\infty}=$ freestream density

$\mu=$ viscosity

$\theta=$ diffuser expansion angle

\section{References}

[1] George, A. R., 1981, "Aerodynamic Effects of Shape Camber, Pitch, and Ground Proximity on Idealized Ground Vehicle Bodies," ASME J. Fluids Eng., 103, pp. 631-638.

[2] George, A. R., and Donis, J. E., 1983, "Flow Patterns, Pressures, and Forces on the Underside of Idealized Ground Effect Vehicles," Proceedings of the ASME, Fluids Engineering Division, 7, ASME, New York, pp. 69-79.

[3] Sovran, G., 1994, "The Kinematic and Fluid-Mechanic Boundary Conditions in Underbody Flow Simulation," Proceedings of the CNR-Pininfarina Workshop on Wind Tunnel Simulation of Ground Effect, Turin, Italy, May.

[4] Cooper, K. R., Bertenyi, T., Dutil, G., Syms, J., and Sovran, G., 1998, "The Aerodynamic Performance of Automotive Underbody Diffusers," SAE Paper No. 980030.

[5] Cooper, K. R., Sovran, G., and Syms, J., 2000, "Selecting Automotive Diffusers to Maximize Underbody Downforce," SAE Paper No. 2000-01-0354.

[6] Senior, A. E., and Zhang, X., 2001, "The Force and Pressure of a DiffuserEquipped Bluff Body in Ground Effect," J. Fluid Eng., 123, pp. 105-111.

[7] Senior, A. E., 2002, "An Investigation of a Generic 3D Diffuser in Ground Effect," Ph.D. thesis, University of Southampton, Apr.

[8] Moffat, R., 1988, "Describing the Uncertainties in Experimental Results," Exp. Therm. Fluid Sci., 1, pp. 3-17.

[9] Polhamus, E. C., 1971, "Predictions of Vortex-Lift Characteristics by a Leading-Edge Suction Analogy,” J. Aircr., 8(4), pp. 193-199.

[10] Wentz, Jr., W. H., and Kohlman, D. L., 1971, "Vortex Breakdown on Slender Sharp-Edged Wings," J. Aircr., 8(3), pp. 156-161.

[11] Lambourne, N. C., and Bryer, D. W., 1962, "The Bursting of Leading-Edge Vortices-Some Observations and Discussion of the Phenomenon," Aeronautical Research Council, Reports and Memoranda No. 3282.

[12] Zerihan, J., and Zhang, X., 2000, “Aerodynamics of a Single Element Wing in Ground Effect," J. Aircr., 37(6), pp. 1058-1064.

[13] Burgin, K., Addey, P. C., and Beatham, J. P., 1986, "Wind Tunnel Tests on Road Vehicle Models Using a Moving Belt Simulation of Ground Effect," J. Wind. Eng. Ind. Aerodyn., 22, pp. 227-236. 